\title{
Percepção da qualidade de vida em crianças e adolescentes usuárias de próteses auditivas
}

\author{
Perception of quality of life in children and \\ teenagers hearing aids users
}

\section{Percepción de la calidad de vida en niños y adolescentes usuarios de prótesis auditivas}

\author{
Renata de Quadros Machado* \\ Laíza Pinheiro Chagas* \\ Eduarda Pazini* \\ Ângela Leusin Mattiazzi* \\ Themis Maria Kessler* \\ Eliara Pinto Vieira Biaggio*
}

\section{Resumo}

Introdução: Com o avanço da tecnologia, os dispositivos eletrônicos relacionados com a reabilitação auditiva suprem de algum modo as necessidades dos usuários e melhoram a qualidade de vida e as habilidades comunicativas. Qualidade de Vida Relacionada à Saúde (QVRS), por ser um tema subjetivo de difícil mensuração, requer a utilização de questionários para tal aferição. Objetivo: Analisar e comparar a percepção de qualidade de vida de crianças e adolescentes usuárias de próteses auditivas na sua visão e de seus familiares/responsáveis, correlacionando-as com as características audiológicas e escolares. Método: Estudo transversal, quantitativo e descritivo, com entrevista estruturada e questionário. Amostra constituída por 25 sujeitos (crianças e adolescentes), idade entre 08 a 18 anos incompletos, diagnosticados com deficiência auditiva bilateral neurossensorial. Os procedimentos utilizados: anamnese; verificação das próteses auditivas; atualização dos dados audiológicos e o questionário Pediatric Quality of Life InventoryTM (PedsQL) versão 4.0 - Português - Brasil, para análise da percepção da QVRS de crianças /adolescentes e seus familiares/responsáveis. Conclusão: Sujeitos com diagnóstico de deficiência auditiva

* Universidade Federal de Santa Maria, Santa Maria, RS, Brasil

Contribuição dos autores:

RQM, LPC, EP - Concepção do estudo, metodologia, coleta de dados, esboço do artigo; ALM, EPVB - Revisão crítica e orientação; TMK- Concepção do estudo, revisão crítica e orientação.

E-mail para correspondência: Renata de Quadros Machado - renata_qmachado@hotmail.com Recebido: $15 / 04 / 2019$

Aprovado: 04/12/2019 
neurossensorial apresentaram semelhante percepção de QVRS comparada às respostas de seus familiares/ responsáveis. As variáveis analisadas no perfil de uso da prótese auditiva não mostraram relação na QVRS dos sujeitos surdos. O grupo dos adolescentes evidenciou uma autopercepção de QVRS inferior à percebida pela faixa etária pediátrica.

Palavras-chave: Qualidade de vida; Inquéritos e questionários; Deficiência auditiva.

\section{Abstract}

Introduction: With the advancement of technology, electronic devices related to hearing rehabilitation somehow fulfill the needs of users and improve quality of life and communication skills. Healthrelated quality of life (HRQOL), it is a subjective theme of difficult measurement, requires the use of questionnaires for such measurement. Objective: To analyze and compare the perception of quality of life of children and adolescents who use hearing aids in their point of view and of their relatives/guardians, correlating them with the audiological and school characteristics. Method: Cross-sectional, quantitative and descriptive study, with structured interview and questionnaire. Sample was composed of 25 subjects (children and adolescents), aged between 08 and 18 years of age, diagnosed with bilateral sensorineural hearing loss. The procedures used: Anamnesis; verification of hearing aids; (PedsQL) version 4.0 Portuguese - Brazil, to analyze the perception of HRQOL of children/adolescents and their relatives/ caregivers. Conclusion: Subjects with sensorineural hearing loss diagnosis presented a similar perception of HRQOL compared to their parents/guardians' answers. The analyzed variables in the hearing aid use profile did not demonstrate being associated to HRQOL of the deaf subjects. The group of adolescents suggested a lower self-perception of HRQOL than the pediatric age group.

Keywords: Quality of Life; Surveys and questionnaires; Hearing loss.

\section{Resumen}

Introducción: Con el avance de la tecnología, los dispositivos electrónicos relacionados con la rehabilitación auditiva suplen de alguna manera las necesidades de los usuarios y mejoran la calidad de vida y las habilidades comunicativas. Calidad de Vida Relacionada a la Salud (QVRS), por ser un tema subjetivo y de difícil mensuración, requiere la utilización de cuestionarios para tal verificación. Objetivo: Analizar y comparar la percepción de calidad de vida en niños y jóvenes usuarios de prótesis auditivas, correlacionándola con los rasgos audiológicos y escolares. Método: Estudio transversal, cuantitativo y descriptivo, con entrevista estructurada y cuestionario. Amuestra constituida por 25 sujetos (niños y adolescentes), edad entre 08 y 18 años incompletos, diagnosticados con pérdida auditiva bilateral neurosensorial. Los procedimientos utilizados: Anamnesis; verificación de las prótesis auditivas; actualización de los datos audiológicos y el cuestionario Pediatric Quality of Life InventoryTM (PedsQL) versión 4.0 - Portugués - Brasil, para el análisis de la percepción da QV de niños/adolescentes y sus familiares/responsables. Conclusión: Sujetos con diagnóstico de pérdida auditiva neurosensorial presentaron semejante percepción de QV comparada a las respuestas de sus familiares o responsables. Las variables analizadas en el perfil de uso de la prótesis auditiva no mostraron relación en la QVRS de los sujetos sordos. El grupo de los adolescentes evidenció una autopercepción de QVRS inferior a la percibida por la franja etaria pediátrica.

Palabras claves: Calidad de Vida; Encuestas y cuestionarios; Pérdida auditiva. 


\section{Introdução}

A audição é um sentido que desempenha um papel relevante para o desenvolvimento das habilidades comunicativas orais, de aprendizagem e de interação social. Neste sentido, a deficiência auditiva (DA) pode gerar uma desarmonia nas habilidades auditivas, ou a inabilidade delas, afetando o desempenho da linguagem oral, impactando diretamente na qualidade de vida do sujeito e comprometendo seu desenvolvimento enquanto ser biopsicossocial ${ }^{1}$.

Historicamente, os sujeitos que tinham diagnóstico de deficiência auditiva neurossensorial de grau severo e/ou profundo bilateral tinham possibilidades muito limitadas para a comunicação oral, bem como para ouvir, o que acarretava efeitos negativos nas suas atividades sociais. Com o avanço da tecnologia, os dispositivos eletrônicos, que estão cada vez mais sofisticados, e a associação com a reabilitação auditiva permitem suprir as necessidades das pessoas com deficiência auditiva, no ensejo de melhorar as suas habilidades comunicativas e, consequentemente, a qualidade de vida desta população².

Cada deficiência envolve diferentes situações sociais e físicas com níveis variados de limitações em termos de atividades diárias e restrição na participação social. As famílias e/ou prestadores de cuidados de saúde também podem ser afetados, dependendo de cada caso ${ }^{3}$.

Sendo a Qualidade de Vida Relacionada à Saúde (QVRS) um tema subjetivo, e de difícil mensuração, a Organização Mundial de Saúde (OMS) a definiu como a percepção do sujeito frente ao contexto cultural e de sistemas de valores, nos quais estão inseridos, em contrapartida aos seus objetivos, expectativas, padrões e preocupações ${ }^{4}$. Com essa definição passou-se a pensar em questionários que permitissem melhor identificar a percepção da qualidade de vida, como uma forma de transformar o que seria subjetivo em objetivo, e analisar o quanto isso afeta a vida de um sujeito.

Aspectos relacionados a questões sociais e emocionais são difíceis de serem avaliados, uma vez que esses aspectos podem variar conforme o dia e o momento que são aplicados. Entretanto, a QVRS é de extrema importância e ignorá-la seria negligenciar etapas do desenvolvimento do sujeito 5 .

A aplicação de questionários possibilita melhores direcionamentos terapêuticos e comparações intra e interpessoais mais concretas, permitindo de tal modo uniformizar diferentes visões ${ }^{6}$. Além disso, a utilização de instrumentos objetivos que incluem a auto percepção, e a auto avaliação de todos os fatores envolvidos é considerada uma opção rápida e eficaz para a investigação do bem-estar do sujeito ${ }^{7}$.

Dentre os questionários destaca-se o Pediatric Quality of Life Inventory (PedsQL) versão 4.0, apropriado para avaliar a qualidade de vida de crianças saudáveis e/ou com doenças crônicas ${ }^{8}$ que foi traduzido e validado para o português brasileiro em $2008^{9}$. O questionário PedsQL foi aplicado em diferentes populações ${ }^{10-11-12-13}$.

Foram encontrados na literatura consultada apenas três estudos com aplicação do questionário QVRS PedsQL versão 4.0 na população com $\mathrm{DA}^{14-15-16}$. Os autores ${ }^{14}$ realizaram a aplicação do instrumento em crianças com DA neurossensorial de grau leve ou moderado, e crianças sem tal déficit sensorial, para analisar a qualidade de vida relacionada à saúde. Outros pesquisadores investigaram a qualidade de vida relacionada à saúde somente em crianças com DA unilateral ${ }^{15}$. Também, foi investigado o efeito da DA nos relatos subjetivos de fadiga, para crianças em idade escolar, utilizando uma medida padronizada ${ }^{16}$.

Tal lacuna identificada na literatura, no que tange à escassez de estudos da mensuração da QVRS na população com DA, reforça a relevância de melhor conhecer a percepção da qualidade de vida de crianças e adolescentes com DA e como seus pais e/ ou cuidadores percebem este aspecto, levando em consideração o perfil de uso das próteses auditivas e outras variáveis.

Portanto, o objetivo deste estudo foi analisar e comparar a percepção de qualidade de vida de crianças e adolescentes usuárias de próteses auditivas na sua visão e de seus familiares/responsáveis, correlacionando-as com as características audiológicas e escolares.

\section{Método}

Estudo do tipo transversal, quantitativo e descritivo, com uso de entrevista estruturada e questionário. Foram observadas as normas da Resolução 466/2012 do Conselho Nacional de Saúde-CNS. Todos os sujeitos assinaram o Termo de Consentimento Livre e Esclarecido (TCLE) e Assentimento. O estudo está vinculado a um 
projeto de pesquisa registrado institucionalmente sob o número 046494, "Desempenho Auditivo e Percepção da Qualidade de Vida em Crianças e Adolescentes Usuárias de Próteses Auditivas", com CAAE 74028617.0.0000.5346 e parecer de aprovação número 2.316.749. A pesquisa foi desenvolvida em uma clínica escola e realizada no período de março a dezembro de 2017.

A amostra foi constituída por 25 sujeitos (crianças e adolescentes), 13 do sexo masculino e 12 do sexo feminino, com idade entre oito a 18 anos incompletos, considerando o que prevê o Estatuto da Criança e do Adolescente (ECA) ${ }^{17}$, diagnosticados com deficiência auditiva bilateral neurossensorial, de grau leve a profundo, usuárias de prótese auditiva, protetizados em serviço de saúde auditiva, via Sistema Único de Saúde (SUS) e seus respectivos familiares. Foram excluídos do estudo sujeitos que não faziam mais o uso da prótese auditiva, que apresentavam alterações de orelha média no momento da realização dos procedimentos para composição amostral, portadores de malformações congênitas e alterações neurológicas e/ou sindrômicas observáveis.

Os procedimentos realizados para a composição do grupo amostral foram: anamnese, com perguntas relacionadas às características escolares e auditivas, verificação das próteses auditivas e atualização dos dados audiológicos para os sujeitos com audiometria tonal liminar realizada há mais de seis meses da data de coleta. Para atualização dos dados audiológicos realizou-se: inspeção visual do meato acústico externo; audiometria tonal liminar seguindo a classificação de Lloyd e Kaplan (1978) ${ }^{18}$ para determinar os limiares auditivos por via aérea nas frequências de 0,25 a $8 \mathrm{kHz}$ e por via óssea nas frequências de 0,5 a $4 \mathrm{kHz}$ em cabina acusticamente tratada segundo a norma ANSI S3.1-1991 de nível de ruído ambiental; logoaudiometria, para determinar o Limiar de Reconhecimento de Fala (LRF) e o Índice Percentual de Reconhecimento de Fala (IPRF) e imitanciometria para análise das condições de orelha média.

Após a realização destes procedimentos, o grupo amostral foi composto por 25 sujeitos usuários de próteses auditivas (com média de idade de 12,9 anos) e seus respectivos familiares. No que diz respeito à DA, todas eram do tipo neurossensorial bilateral, com variabilidade do grau entre leve à profundo, sendo três sujeitos apresentando grau leve $(12,00 \%)$, cinco com grau moderado $(20,00 \%)$, nove moderadamente severo $(36,00 \%)$, seis severo $(24,00 \%)$ e dois profundo $(8,00 \%)$.

Como procedimento de pesquisa os sujeitos foram convidados a responder o questionário PedsQL versão 4.0. - Português - Brasil, com auxílio da leitura do pesquisador quando necessário. Os sujeitos foram orientados a considerar as experiências do último mês, para as respostas, como indicam as instruções de aplicabilidade do teste. Neste estudo foram utilizados dois módulos do questionário PedsQL, um aplicado aos familiares/responsáveis e outro aos sujeitos com DA levando-se em consideração a idade cronológica de cada sujeito para a escolha da entrevista. As versões que compõem o questionário para cada faixa etária - 8-12 anos e 13-18 anos - são equivalentes, diferindo apenas quanto à terminologia em função do grau de desenvolvimento esperado em cada idade. A aplicação dos questionários aos familiares/ responsáveis foram no período em que a criança/adolescente estava realizando os procedimentos audiológicos, em uma sala de terapia disponível, após então, aplicado nos sujeitos com DA na sala de avaliação.

O questionário PedsQL 4.0 constitui-se de formulários de autoavaliação dirigidos à crianças/ adolescentes e questionários aos pais referentes à percepção destes com relação à qualidade de vida do filho. Em ambos os formulários, foram utilizados pelos sujeitos a escala de respostas de zero a quatro níveis. Zero correspondente a nunca ser um problema; um correspondente a quase nunca ser um problema; dois corresponde a algumas vezes ser um problema; três muitas vezes ser um problema; quatro quase sempre ser um problema.

O questionário foi analisado por meio do resultado total e de dois sub-resultados relacionados à saúde física que possui oito itens, e psicossocial com 15 itens. A análise do questionário considerou o escore antagônico e linear na pontuação dos itens, transformado em uma escala que corresponde de zero (0) a cem (100), ou seja, zero é substituído por 100 , um para 75 , dois para 50 , três para 25 , quatro para zero.

Os escores totais, o psicossocial e a capacidade física foram computados pela soma dos itens dividida pelo número de itens respondidos. Assim, quanto maior o escore, melhor a qualidade de vida relacionada à saúde.

Os resultados obtidos de todos os sujeitos foram analisados e pareados com as informações 
colhidas no momento da anamnese e nos procedimentos audiológicos realizados no estudo.

As variáveis analisadas foram: escores no questionário PedsQL 4.0 obtidos pelas crianças/ adolescentes e seus respectivos familiares/responsáveis, grau de DA, DA progressiva ou não, efetividade de uso das próteses auditivas (sendo considerado uso efetivo oito horas ou mais ${ }^{19}$ ), uso do Sistema FM e o tipo de escola frequentada pelos sujeitos (regular ou especial).

Todos os dados coletados foram transcritos em uma tabela no programa Excel (2010), visando melhor visualização e para posterior análise estatística.

Os dados foram submetidos à análise estatística descritiva e analítica, a partir dos testes de compara- ção de Wilcoxon, Mann-Whitney e Teste de Correlação de Spearman em nível de significância de 0,05 com intervalos de $95 \%$ de confiança estatística.

\section{Resultados}

Em relação ao questionário PedsQL 4.0, foi realizada a análise global dos escores obtidos por subgrupos de crianças/adolescentes e de seus respectivos familiares/responsáveis.

Assim, compararam-se os valores das respostas dos sujeitos entre os escores obtidos pelos familiares/responsáveis, podendo ser observado na Tabela 1.

Tabela 1. Escores, por categoria, do questionário de Qualidade de Vida - Pediatric, Quality, of Life Inventory TM 4.0 (Peds-QL TM) e comparação entre os escores obtidos dos sujeitos e dos familiares/ responsáveis $(n=X X)$

\begin{tabular}{lccc}
\hline \multirow{2}{*}{ QUALIDADE DE VIDA } & SUJEITO & RESPONSÁVEL & \multirow{2}{*}{ Valor de p } \\
\cline { 2 - 3 } & Md (Xmín; Xmáx) & Md (Xmín; Xmáx) & \\
\hline Capacidade Física & $75,0(34,4 ; 100,0)$ & $87,5(28,1 ; 100,0)$ & 0,6186 \\
Aspecto Emocional & $65,0(20,0 ; 100,0)$ & $70,0(25,0 ; 100,0)$ & 0,2604 \\
Aspecto Social & $75,0(15,0 ; 100,0)$ & $75,0(25,0 ; 100,0)$ & 0,8638 \\
Aspecto Escolar & $65,0(35,0 ; 90,0)$ & $60,0(20,0 ; 100,0)$ & 0,0666 \\
Aspecto psicossocial & $73,3(25,0 ; 88,3)$ & $66,7(30,0 ; 88,3)$ & 0,6570 \\
\hline Total dos Escores & $72,9(28,3 ; 89,1)$ & $74,0(35,9 ; 91,3)$ & 0,6265 \\
\hline
\end{tabular}

Legenda: Md = mediana; XMin. = valor mínimo; XMax. = valor máximo;

Teste de U de Mann-Whitney;

Esta primeira análise foi realizada de forma global, em que as respostas de todos os sujeitos e todos familiares/responsáveis foram comparadas para possível significância.
Na Tabela 2, foram cruzados a mensuração da QVRS obtidos nas categorias do Peds-QL, com o grau de deficiência auditiva.

Tabela 2. Comparação das pontuações pelos escores nas categorias do Pediatric Qualityof Life Inventory TM 4.0 (Peds-QL TM) com o grau da deficiência auditiva $(n=X X X)$

\begin{tabular}{lcc}
\hline & Grau da Deficiência Auditiva & \\
\hline Variáveis & $\mathbf{r}$ & Valor de p \\
\hline Capacidade Física & $-0,234048$ & 0,260141 \\
Aspecto Emocional & 0,147418 & 0,481924 \\
Aspecto Social & $-0,142929$ & 0,495512 \\
Aspecto Escolar & 0,005612 & 0,978762 \\
Aspecto Psicossocial & $-0,04815$ & 0,819213 \\
\hline TOTAL & $-0,080623$ & 0,701645 \\
\hline
\end{tabular}

Teste de Correlação de Spearman 
Na Tabela 3, mostra-se a comparação das pontuações pelos escores obtidos das categorias do Peds-QL TM, entre as variáveis: DA quanto à característica de ser progressiva ou não; efetividade de uso das próteses auditivas; uso do Sistema FM e o tipo de escola frequentada pelos sujeitos, se regular ou especial.

$\mathrm{Na}$ Tabela 4, foram analisadas a percepção dos familiares dos dois subgrupos e dos sujeitos-crianças/adolescentes.

Tabela 3. Comparação das pontuações dos escores obtidos nas categorias do Pediatric Qualityof Life Inventory TM 4.0 (Peds-QL TM) entre as variáveis deficiência auditiva, efetividade de uso das próteses auditivas, uso ou não do Sistema de Frequência Modulada e o tipo de escola

\begin{tabular}{|c|c|c|c|c|c|c|c|}
\hline & & \multicolumn{6}{|c|}{ Qualidade de Vida Md (Xmáx; Xmín) } \\
\hline & & $\mathbf{C F}$ & $\overline{\mathrm{AE}}$ & ASO & AESC & PSICO & TOTAL \\
\hline Perda & ogr &, $0(40,6 ; 75,0)$ &, $0(40,0 ; 75$, & ) & 4 & 7) & ras \\
\hline Aud & & $\begin{array}{l}0(3 \\
0,\end{array}$ & $\begin{array}{c}70,0(20,0 ; 10 \\
0,0738 \\
\end{array}$ & $\begin{array}{r}80,0(1 \\
0 \\
\end{array}$ & $\begin{array}{r}70,0(3 \\
0,\end{array}$ & $\begin{array}{l}\text {;8,9) } \\
2^{*}\end{array}$ & $\begin{array}{r}, 0(2 \\
0,(\end{array}$ \\
\hline Uso & $\mathrm{p}$ & $75,0(34,3$ & $65,0(20$, & $\begin{array}{l}0,0) \\
5,0)\end{array}$ & $\begin{array}{l}90,0) \\
85,0) \\
6\end{array}$ & $\begin{array}{r}76,7(25 \\
60,0(4 \\
0,1\end{array}$ & $\begin{array}{c}76,0(28,2 ; 89 \\
62,4(41,3 ; 83 \\
0,161\end{array}$ \\
\hline & & $(40,6 ; 100,0)$ & $(20,0 ; 100,0)$ & $0(3$ & $70,0(40,0 ; 90,0)$ & $7(41,7 ; 83,3)$ & $3 ; 8$ \\
\hline & & $\begin{array}{r}3(34 \\
0,3 \\
\end{array}$ & $\begin{array}{c}5(25,0 ; 8 \\
0,4351\end{array}$ & $\begin{array}{l}0(1 \\
0,\end{array}$ & 0 , & $\begin{array}{l}3(25,0 ; 88,3) \\
0,113\end{array}$ & 0,1567 \\
\hline $\begin{array}{l}\text { Tip } \\
\text { Es }\end{array}$ & $\begin{array}{l}\text { Especial } \\
\text { p- valor }\end{array}$ & $\begin{array}{c}75,0(34,3 ; 100,0) \\
65,6(40,6 ; 100,0) \\
0,738\end{array}$ & $\begin{array}{c}60,0(20,0 ; 85,0) \\
80,0(40,0 ; 100,0) \\
0,2482\end{array}$ & $\begin{array}{c}80,0(15,0 ; 100,0) \\
60,0(40,0 ; 90,0) \\
0,3331\end{array}$ & $\begin{array}{c}70,0(35,0 ; 90,0) \\
57,5(40,0 ; 90.0) \\
0,5761\end{array}$ & $\begin{array}{c}73,3(25,0 ; 83,3) \\
67,5(42,0 ; 88,2) \\
0,9407\end{array}$ & $\begin{array}{c}73,0(28,2 ; 89,1 \\
71,0(41,3 ; 85,0 \\
0,9407\end{array}$ \\
\hline
\end{tabular}

Legenda: * Valor estatisticamente significante $(p \leq 0,05) \cdot D A=$ Deficiência auditiva; $F M=$ Frequência Modulada; Md=mediana; $X M i n .=$

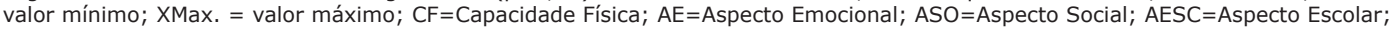
APSICO =Aspecto Psicossocial.

**Teste U de Mann-Whitney

Tabela 4. Comparação das pontuações pelos escores obtidos nas categorias do Pediatric Qualityof Life Inventory TM 4.0 (Peds-QL TM) entre a percepção dos familiares/responsável versus crianças/ adolescentes $(\mathrm{n}=\mathrm{XX})$

\begin{tabular}{lcccccc}
\hline \multirow{2}{*}{ Variáveis } & \multicolumn{2}{c}{ Md (Xmáx; Xmín) } & \multirow{2}{*}{ Valor de p } & \multicolumn{2}{c}{ Md (Xmáx; Xmín) } \\
\cline { 2 - 3 } & Pais grupo 8-12 a & Pais grupo 13-18 a & & crianças 8-12 a & Adolescentes13-18a & Valor de p \\
\hline CF & $59,4(100,28,1)$ & $89,1(96,9 ; 46,9)$ & 0,722958 & $75(100 ; 40,7)$ & $70,3(100 ; 34,4)$ & 0,140405 \\
AE & $70(100 ; 25)$ & $72,5(95 ; 50)$ & 0,956412 & $60(100 ; 20)$ & $65(85 ; 25)$ & 0,259165 \\
ASO & $50(100 ; 25)$ & $87,5(100 ; 35)$ & 0,512396 & $75(100 ; 35)$ & $77,5(100 ; 15)$ & $72,5(90 ; 35)$ \\
AESC & $40(85 ; 20)$ & $77,5(100 ; 35)$ & 0,460339 & $65(90 ; 40)$ & 0,003204 \\
PSICO & $55(86,7 ; 30)$ & $79,2(88,3 ; 61,7)$ & 0,913173 & $76,1(88,3 ; 41,7)$ & $72,5(83,3 ; 25)$ & 0,002509 \\
TOTAL & $53,3(90,2 ; 35,9)$ & $83,2(91,3 ; 57,6)$ & 0,934898 & $76,1(90,2 ; 35,9)$ & $69,6(89,1 ; 28,3)$ & 0,016505 \\
\hline
\end{tabular}

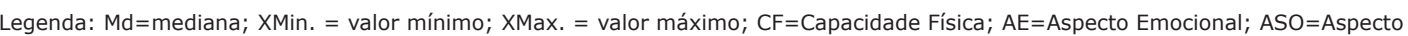
Social; $\mathrm{AESC}=$ Aspecto Escolar; $\mathrm{APSICO}=$ Aspecto Psicossocial. Teste $\mathrm{U}$ de Mann-Whitney.

\section{Discussão}

Em relação à análise do escore total do questionário PedsQL 4.0, 23 sujeitos apresentaram uma adequada QVRS, com pontuação acima de $50 \%$ e dois sujeitos foram classificados com baixa QVRS. Na presente pesquisa, todos os participantes realizam/realizaram acompanhamento no setor de Reabilitação auditiva, o que inclui terapia fonoaudiológica e revisões periódicas das próteses auditivas. Dessa maneira, os sujeitos têm um auxílio do profissional fonoaudiólogo quando necessário, contribuindo assim, para uma melhor percepção da deficiência auditiva, suas limitações e possíveis soluções, o que contribui para uma melhor qualidade de vida.

Conforme o exposto na Tabela1, não foi observada diferença estatisticamente significante entre as percepções do sujeito com deficiência auditiva em relação à dos seus familiares/responsáveis 
sobre a QVRS. Chama atenção o item da percepção dos aspectos escolares, em que os familiares/ responsáveis avaliaram a QVRS de seus filhos com uma percepção inferior quando comparado com a análise que as crianças / adolescentes fizeram de si mesmos, ou seja, os sujeitos (crianças / adolescentes) apresentaram uma análise positiva de QVRS no aspecto escolar, indicando uma adequada adaptação no ambiente acadêmico, porém sem diferenças estatisticamente significantes. Tais achados mostram semelhanças com outro estudo que utilizou o mesmo questionário (PedsQL) ${ }^{15} \mathrm{e}$ que os pesquisadores aplicaram na segunda etapa do estudo, o instrumento genérico de qualidade de vida (PedsQL) a uma população de normouvintes (composta por 24 crianças), população com deficiência auditiva unilateral (32 crianças) e a uma população com DA bilateral (29 crianças), objetivando mensurar a qualidade de vida relacionada à saúde. $\mathrm{O}$ instrumento foi aplicado aos pais e às próprias crianças. Em sua primeira etapa, revelou que crianças com deficiência auditiva unilateral enfrentam dificuldades, no entanto aprendem a se adaptar à sua condição. Com isso, tanto no questionário aplicado aos pais, quanto na versão aplicada às crianças e adolescentes, o resultado da amostra dos três grupos, mostrou que não houve diferença quantitativamente significante em nenhuma das três escalas física, psicossocial e total do instrumento empregado ${ }^{15}$.

Ao comparar o presente estudo com o estudo supracitado, observa-se que a amostra é razoavelmente maior, mesmo assim, ambos os estudos obtiveram o mesmo resultado quantitativo. Pode-se inferir que os sujeitos com deficiência auditiva juntamente com seus familiares/responsáveis estão se adaptando em seu cotidiano e apresentando uma percepção similar de QVRS nos aspectos avaliados no instrumento.

Em um outro estudo foi aplicado o questionário validado e padronizado, traduzido para Língua de Sinais (HRQoL) para a faixa etária pediátrica e outro para seu membro familiar por meio do questionário KIDSCREEN-27 com o intuito de analisar a QVRS de 114 crianças e adolescentes surdos com diferentes níveis de deficiência auditiva, com idade entre oito e 18 anos, vivendo em áreas rurais e urbanas, e pertencentes tanto ao setor público quanto ao privado. Como resultado os autores não encontraram diferenças entre Índices médios de crianças / adolescentes e seus pais em todas as di- mensões do questionário KIDSCREEN-27, exceto na autonomia e no suporte social ${ }^{20}$.

Outro estudo obteve similaridade nas informações fornecidas por pais e filhos, tal como o ocorrido no presente estudo. Acredita-se que pais/ cuidadores, no momento de mensurarem a QV de seus filhos, responderam à escala como projeção do que a criança/adolescente teria dado como resposta, embora tenham sido orientados de como deveriam responder o questionário. Admitindo-se esta possibilidade, tal interpretação do familiar ao responder ao instrumento, deve ser considerada como um efeito sobre a análise da real percepção do que os familiares/responsáveis inferem ser a QV de seus respectivos filhos/sujeitos ${ }^{21}$.

Como observado na Tabela 2, não houve diferença entre a percepção da qualidade de vida dos sujeitos da amostra e os diferentes graus de DA. Este dado concorda com um estudo ${ }^{14}$, no qual os autores fizeram uma investigação semelhante, porém com uma amostra de 6581 crianças, estudantes do ensino fundamental, parte da amostra dos sujeitos diagnosticados com deficiência auditiva neurossensorial de grau leve a moderado e outra parte da amostra constituída de crianças normo-ouvintes, em que também não se observou diferenças estatisticamente significantes entre as variáveis QVRS, linguagem, leitura e comportamento, porém a habilidade de memória fonológica de curto prazo no grupo de sujeitos com DA neurossensorial apresentou-se rebaixada quando comparada ao grupo de sujeitos normo-ouvintes. Esta análise pressupõe a boa adaptação dos usuários com as próteses auditivas, de modo a interferir positivamente nas categorias avaliadas do instrumento, independentemente do grau do déficit auditivo.

Outro estudo objetivou mensurar a QV em adolescentes com DA e seus irmãos normo-ouvintes por meio de quatro questionários, dentre eles o PedsQL TM 4.0. Como resultados, também não encontraram diferença estatisticamente significante nos resultados da mensuração da QVRS entre os grupos estudados, ou seja, o grau de deficiência auditiva não modificou na percepção de $\mathrm{QVRS}^{22}$.

Cabe ressaltar que os autores do presente acreditavam que crianças e adolescentes com deficiência mais severa apresentariam pior percepção de qualidade de vida, mas tal hipótese não se confirmou. Infere-se que isto aconteceu, por todos os sujeitos da amostra serem pacientes de um serviço de Habilitação e Reabilitação da audição há muito 
tempo e neste serviço contavam com fonoterapia e intervenção psicológica, além de participação em grupos de pais. Estes fatores podem ter influenciado diretamente na percepção de qualidade de vida dos sujeitos do grupo amostral.

No que se refere à informação sobre o uso efetivo das próteses auditivas, obtidas por meio do relato do familiar / responsável, e a variável QVRS (Tabela 3), não houve diferença estatisticamente significante. Em outros estudos, quando comparado o tempo relatado pelos pais, versus o que foi encontrado por análises objetivas, verifica-se que os pais/ cuidadores apresentam tendência em superestimar o tempo de uso da amplificação em cerca de $2 / 5$ horas, principalmente para crianças menores ${ }^{23-24}$.É possível que esta diferença metodológica possa auxiliar a compreensão da ausência de relação do uso com a percepção de QV em nosso estudo, visto que foi somente comparado informação subjetiva do uso das próteses auditivas.

Outra pesquisa, realizada com 272 sujeitos com idade entre cinco meses a sete anos e três meses, com deficiência auditiva de grau leve a severo, buscou estimar o tempo de uso diário das próteses auditivas, de forma subjetiva e objetiva. $\mathrm{O}$ uso foi menos efetivo em sujeitos de menor idade e deficiência auditiva leve quando comparadas às crianças mais velhas e com deficiências severas ${ }^{23-25}$.

Em estudo recente, com uma amostra de 20 sujeitos, em que foi analisada a relação da classificação socioeconômica e a percepção da qualidade de vida, entretanto, com o uso de outro instrumento, e desenvolvido apenas com familiares de crianças e adolescentes com deficiência auditiva, foi observado melhor qualidade de vida do familiar/responsável no domínio social e pior qualidade de vida no domínio ambiental. Com isso, o estudo mostrou que houve relação entre a classificação socioeconômica e a percepção da qualidade de vida dos familiares, fato que não foi correlacionado em nosso estudo, sendo uma lacuna que pode ser analisada por outros pesquisadores da área ${ }^{26}$.

Ainda sobre a Tabela 3, no que diz respeito aos escores das análises das variáveis uso efetivo da prótese auditiva e do sistema FM, bem como o tipo de escola ser regular ou especial, não houve evidência de relação com a QV referida pelos sujeitos. Esse indicador discorda de outro estudo, no qual o uso da prótese auditiva mostrou inúmeros benefícios para os sujeitos principalmente no nível escolar. Mas corrobora quando comparado aos tipos de escolas (regular e especial) que também não mostrou resultados significantes que influenciariam na QVRS ${ }^{21}$. Acredita-se que alguns resultados estatísticos deste estudo foram influenciados pelo tamanho amostral e que na composição amostral quatro sujeitos estudavam em escola especial, utilizando também a língua de sinais, mantendo boas relações sociais, culturais e não prejudicando na qualidade de vida.

Em 2007, um estudo realizado com crianças com deficiência auditiva tardia demonstrou que esses sujeitos tendem a reportar maiores níveis de insatisfação com suas vidas sociais do que sujeitos com deficiência auditiva congênita, pois essa população tende a adquirir a língua de sinais dentro da comunidade surda tardiamente ${ }^{27}$. Na presente amostra, são esses sujeitos que utilizam a língua de sinais em ambientes sociais, que acabam não fazendo o uso efetivo da prótese auditiva, visto que a utilizam apenas em ambiente familiar quando os pais e ou responsáveis assim desejam.

Cabe ressaltar que as análises que mais se aproximaram dos valores de significância estatística foram os escores referentes ao uso da prótese auditiva versus o aspecto social. Os sujeitos que utilizam mais de oito horas apresentam uma melhor percepção de QV no aspecto social, não sendo significativa, porém observado nas diferenças das medianas dos resultados.

Com relação à classificação da deficiência auditiva, progressiva ou não progressiva, também observado na Tabela 3, sujeitos que apresentam DA não progressiva tiveram uma percepção significativamente melhor na QVRS em aspecto psicossocial e no escore total quando comparados aos indivíduos com DA progressiva, o que corrobora com outro estudo $^{27}$. O cruzamento desta variável com o aspecto emocional também se aproximou ao valor de significância (valor de p 0,07).

O uso do sistema FM (Tabela 3) não apresentou significância estatística nas questões de QVRS no aspecto social (valor de p 0,0512) e escolar (valor

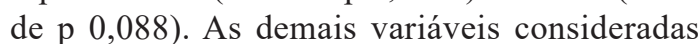
apresentaram um comportamento semelhante do ponto de vista estatístico, sem representar influência direta com a percepção de qualidade de vida, analisada por meio do protocolo PedsQL. Outro estudo ${ }^{16}$ mensurou o efeito da deficiência auditiva em relatos subjetivos de fadiga em crianças em idade escolar utilizando o instrumento Pediatric Quality of Life Inventory (PedsQL) Multidimen- 
sional Fatigue Scale ${ }^{28}$, em uma amostra de 10 crianças diagnosticadas com deficiência auditiva e 10 crianças da mesma idade normo-ouvintes, os sujeitos com DA faziam uso das próteses auditivas ou IC em tempo integral no ambiente escolar e sete sujeitos faziam uso do Sistema FM. O estudo concluiu que crianças com DA em idade escolar relataram significativamente mais fadiga quando comparadas às crianças normo-ouvintes, achados relevantes no desempenho acadêmico e psicossociais associados à fadiga.

Ao analisar a Tabela 4, observa-se que o grupo dos adolescentes obteve uma baixa percepção de QVRS nos aspectos social, escolar, psicossocial e total quando comparado aos escores esperados para a respectiva faixa etária. Verificou-se na literatura um estudo que mensurou a qualidade de vida de crianças alemãs com DA e de crianças ouvintes que estavam inseridas em escola regular, usando o Inventário da Qualidade de Vida de Crianças e Jovens (ILC) $^{29,30}$. Os resultados mostraram que crianças com deficiência auditiva tiveram uma percepção de qualidade de vida significativamente maior do que os estudantes ouvintes e estavam mais satisfeitos com a escola. Os autores verificaram em seu estudo que a população pediátrica (de 8 a 11 anos) obteve pontuações mais elevadas em vários domínios do instrumento supracitado do que os adolescentes (de 12 a 18 anos). Esses escores mais baixos no grupo de adolescentes pode ser uma consequência de uma maior autoconsciência de suas limitações O presente estudo corrobora com os achados dos estudos acima mencionados, ainda que tenham sido utilizados procedimentos diferentes quanto à modalidade linguística de apresentação do instrumento na coleta nesta pesquisa, pois o grupo amostral dos adolescentes também evidenciou escores de QVRS inferiores no instrumento PedsQL.

\section{Conclusão}

Conforme o exposto, foi possível concluir que crianças e adolescentes com deficiência auditiva neurossensorial de grau leve a profundo apresentaram semelhança na percepção de QVRS ao serem comparadas com os escores obtidos pelos seus familiares e/ou responsável, no que se refere à análise geral.

Não houve relação direta na QVRS dos sujeitos quanto ao grau da deficiência auditiva, uso das próteses auditivas, uso do sistema FM e tipo de escola. A variável deficiência auditiva não progressiva correlacionou-se positivamente na QVRS dos sujeitos da amostra, assim como os adolescentes demonstraram possuir uma autopercepção de QVRS significativamente inferior à da sua respectiva faixa etária.

\section{Referências}

1. Calháu CMDF, Júnior LRPL, Reis AMCS, Capistrano AKB, Lima DVSP, Calháu ACDF et al. Etiology profile of the patients implanted in the cochlear implant program. Rev Braz J Otorhinolaryngol. 2011; 77(1): 13-8.

2. Magalhães ATM, Goffi-Gomez MV, Jardim I, Tsuji RK, Neto RB, Bento RF. Adaptação de próteses auditivas no candidato ao implante coclear. Rev CEFAC. 2011; 13(1): 75-84

3. Van der Slot WM, Nieuwenhuijsen C, Berg-Emons VDRJ, Wensink-Boonstra AE, Stam HJ, Roebroeck ME. Participation and health-related quality of life in adults with spastic bilatera cerebral palsy and the role of self-efficacy. Rev J Rehabil Med. 2010; 42(6): 528-35.

4. World Health Organization (WHO). The World Health Organization Quality of Life Assessment (WHOQOL): position paper from the World Health Organization. Rev Soc Sci Med 1995; 41(10): 1403-09.

5. Jorge BM, Levy CCAC, Granato L. Adaptação Cultural da Escala de Qualidade de Vida Familiar (Family Quality of Life Scale) para o Português Brasileiro. Rev CoDAS. 2015; 27(6): 534-40.

6. Landeiro GMB, Pedrozo CCR, Gomes MJ, Oliveira ERA Revisão sistemática dos estudos sobre qualidade de vida indexados na base de dados. Cien Saude Colet. 2011; 16(10): 4257-66.

7. Bowling A. Mode of questionnaire administration can have serious effects on data quality. Rev J Public Health (Oxf). 2005; 27(3): 281-91.

8. Varni JW, Seid M, Rode CA. The PedsQL ${ }^{\mathrm{TM}}$ : Measurement model for the pediatric quality of life inventory. Med Care. 1999; 37 (2) 126-39.

9. Klatchoian DA, Len CA, Terreri MTRA, Silva M, Itamoto C, Ciconelli RM et al. Qualidade de vida de crianças e adolescentes de São Paulo: confiabilidade e validade da versão brasileira do questionário genérico Pediatric Quality of Life InventoryTM versão 4.0. J Pediatr (Rio J). 2008; 84 (4): 308-15.

10. Neder L, Weeldenb MV, Viola GR, Lourenço DM, Claudio $\mathrm{AL}$, Lene $\mathrm{E}$, et al. Qualidade de vida relacionada à saúde avaliada pelo Inventário Pediátrico de Qualidade de Vida 4.0 em pacientes pediátricos com hanseníase e manifestações musculoesqueléticas. Rev bras reumatol. 2015; 55(5): 414-19.

11. Macedo RB, Silva MJC, Sousa NF, Santos JV, Rodrigues AMM, Cumming SP, et al. Quality of life, school backpack weight, and nonspecific low back pain in children and adolescents. Rev J Pediatr. 2015; 91(3): 263-9.

12. Araújo J, Dourado M, Ferreira PL. Instrumentos de Medição da Qualidade de Vida em Idade Pediátrica em Cuidados Paliativos. Rev Acta Med Port. 2015; 28(4): 501-12. 
13. Abreu IS, Santos CB. Impacto da insuficiência renal crônica na qualidade de vida de crianças e adolescentes: revisão integrativa. Rev. eletrônica enferm. 2014; 16(4): 833-41.

14. Wake M, Tobin S, Cone-wesson B, Dahl HH, Gillam L, Mccormick L et al. Slight / Mildsensorineural hearing loss in children. Rev Pediatrics. 2006; 118(5): 1842-51.

15. Borton SA, Mauze E, Lieu JEC. Quality of life in children with unilateral hearing loss: apilot study. Rev J Audiol 2010; 19(1): 61-72.

16. Hornsby BW, Werfel K, Camarata S, Bess FH. Subjective fatigue in children with hearing loss: Some preliminary findings. Rev J Audiol. 2014; 23 (1):129-34.

17. Brasil. Estatuto da criança e do adolescente. 9. ed. Brasília: Diário Oficial da União. Câmara editora; 2012.

18. Lloyd LL, Gladstone V, Kaplan H. Audiometric interpretation: manual of basic audiometry. 2 ed. Massachusetts: Pearson; 1993.

19. Luz VB, Silva MC, Scharlach RC, Iório MCM. Correlação entre as restrições de participação em atividades de vida diária e o benefício do uso de próteses auditivas em adultos e idosos. Rev Soc Bras Fonoaudiol. 2011; 16(2): 160-6.

20. Pardo-guijarro MJ, Martínez-andres M, Notario- Pacheco B, Solera-martinez M, Sánchez-lopez M, Martínez-vizcaíno V. Self-Reports Versus Parental Perceptions of Health- Related Quality of Life Among Deaf Children and Adolescents. J Deaf Stud Deaf Educ. 2015; 20 (3): 275-82.

21. Carvalho LRL, Lichtig I, Couto MIV. Avaliação do benefício do uso de aparelhos de amplificação sonora individual em crianças. Rev Otorhinolaryngol. 2012;16(2): 170-8.

22. Rachakonda T, Jeffe DB, Shin JJ, Mankarious L, Fanning RJ, Lesperance MM, et al. Validity, Discriminative Ability and Reliability of the Hearing- Related Quality of Life (HEARQL) Questionnaire for Adolescents. Rev Laryngoscope. 2014; 124(2): 570-8.
23. Walker EA, Spratford M, Moeller MP, Oleson J, Roush P, Jacobs S. Predictors of hearing aid use time in children with mild-to-severe hearing loss. Rev Lang Speech Hear Serv Sch. 2013; 44 (1): 73-88.

24. Muñoz K, Preston E, Hicken S. Pediatric hearing aid use: How can audiologists support parents to increase consistency? Rev J Am Acad Audiol. 2014; 25(4): 380-7.

25. Tomblin JB, Oleson JJ, Ambrose SE, Walker E, Moeller MP. The Influence of Hearing Aids on the Speech and Language Development of Children With Hearing Loss. Rev Otolaryngol Head Neck Surg. 2014;140(5): 403-9.

26. Nascimento GB, Schiling NO, Ubal SR, Biaggio EPV, Kessler TM. Classificação socioeconômica e qualidade de vida de familiares de crianças e adolescentes com deficiência auditiva. Rev CEFAC. 2016; 18(3): 657-66.

27. Fellinger J, Holzinger D, Gerich J, Goldberg, D. Mental distress and quality of life in the hard of hearing. Rev Acta Psychiatr Scand. 2007; 115 (3): 243-5.

28. Varni JW, Seid M, Kurtin PS. The PedsQL 4.0: reliability and validity of the Pediatric Quality of Life Inventory 4.0 $0^{\mathrm{TM}}$ version 4.0 Generic Core Scales in Healthy and Patient Populations. Med Care. 2001; 39(8) 800-12.

29. Hintermair M. Health-related quality of life and classroom participation of deaf and hard-of-hearing students in general schools. J Deaf Stud Deaf Educ. 2011; 16(2): 254-70.

30. Schick B, Skalicky A, Edwards T, Kushalnagar P,Topolski T, Patrick D. School Placement and Perceived Quality of Life in Youth Who Are Deaf or Hard of Hearing. J Deaf Stud Deaf Educ.. 2013; 18(1): 47-61. 